\title{
Status of the EDELWEISS II experiment
}

\section{Xavier DEFAY for the EDELWEISS collaboration}

CSNSM

Orsay, France

E-mail: defayecsnsm.in2p3.fr

EDELWEISS is a direct dark matter search located in the low radioactivity environment of the Modane Underground Laboratory. The experiment uses massive Ge detectors at very low temperature in order to identify eventual rare nuclear recoils induced by elastic scattering of WIMPs from our galactic halo. The commissioning of the second phase of the experiment, involving more than $7 \mathrm{~kg}$ of Ge detector, has been completed in 2007. These ionization/heat detectors allow an event by event electronic/nuclear recoil discrimination. A complementary active rejection of surface events is required to achieve the physics goals of the present phase. We present preliminary results of detectors with active rejection (new type of bolometer with interdigitized electrodes for the charge collection). 


\section{Introduction}

The EDELWEISS experiment is a direct dark matter search located in the low radioactive background of the Modane Underground Laboratory (LSM). The direct detection principle is to detect the energy deposited by the elastic scattering of a WIMP (of a typical mass between few tens and few hundreds of $\left.\mathrm{GeV} / \mathrm{c}^{2}\right)$ from the galactic halo on target nuclei. The main difficulty comes from the low rate of interaction which is lower than one event per hundred kilograms per day with a relatively small energy deposited $(<100 \mathrm{keV})$.

\section{Principle of the experiment and main limitation}

The detectors used in EDELWEISS are cryogenic high purity germanium bolometers with double component ionization and heat. The ionization charges produced by the interaction of a particle are collected by applying an electric field with electrodes at each side of the crystal. The signal is read by charge sensitive amplifiers. The rise in temperature is measured by a Neutron Transmutation Doped (NTD) thermometer which resistance changes with temperature.

The ionization signal depends on the type of particle whereas the heat signal is function of the energy deposited by any particle in the crystal. Comparing the two simultaneous measurements (heat and ionization) provides an event by event discrimination between nuclear recoils (like wimps and neutrons) and electron recoils (induced by photons and electrons) which is the dominant background.

To increase its sensitivity to the WIMP, the experiment has to increase the detection mass and to reduce the radioactive background sources $^{1}$ that have limited the EDELWEISS I experiment ${ }^{2}$.

EDELWEISS II has been improved looking the radiopurity ${ }^{3}$. A systematic check of all material radiopurity installed near the detectors has been operated with germanium detector specially dedicated for this. The cryostat has been installed in a clean room with deradonized air $\left(<0.1 \mathrm{~Bq} \cdot \mathrm{m}^{-3}\right)$. The gamma background is screened by a lead shield of a thickness of $20 \mathrm{~cm}$.

The neutron shield has been thickened to $50 \mathrm{~cm}$ and a muon-veto allows to reject neutron events induced by muons by tagging events in coincidence between the muon-veto and the detectors. The rate of remaining muon-induced nuclear recoils (with an energy deposited $>10$ $\mathrm{keV}$ ) computed by Monte-Carlo simulations is lower than $10^{-4}$ neutron recoils per kilogram per day $^{4}$, which does not limit the EDELWEISS WIMP-nucleon cross section sensitivity down to $\sim 10^{-9} \mathrm{pb}$ for a WIMP of $100 \mathrm{GeV}$, improving the sensitivity by more than a factor 100 compared to the EDELWEISS I experiment.

Lastly, tests are underway to integrate a scintillation-phonon detector into EDELWEISS $\mathrm{II}^{5}$. This could expand the range of target nuclei available for the experiment, and provide information on backgrounds.

Moreover, the cryostat capacity has been raised from $1 \mathrm{~kg}$ of detector in EDELWEISS I to $40 \mathrm{~kg}$ of detector in the EDELWEISS II experiment.

The main limiting background of the experiment comes from events occurring in the vicinity of the surfaces of the detector near the collecting electrodes (essentially beta event from the ${ }^{210} \mathrm{~Pb}$ contamination in the vicinity of the detectors). These events have a 
bad charge collection that can mimic nuclear recoil and limit the sensitivity of the experiment.

Two types of detectors with surface event rejection capabilities have been tested: $400 \mathrm{~g} \mathrm{NbSi}$ replacing the NTD thermistors by two NbSi thin films sensors which are sensitive to the athermal part of the phonon signal ${ }^{6,7}$ (allowing a surface event with the phonon signal) and Ge/NTD crystal with interdigitized electrodes. We will focus here on the second type of detector.

\section{Surface event rejection with interdigitized electrodes}

The new bolometer Interdigit ${ }^{8,9}$ is based on the ionization signals to reject surface events. It's a cylinder of germanium with two interleaved electrodes at the top face, two at the bottom and two guard rings to reject events coming from the sides of the cristal. The phonon signal is read by an NTD thermometer.

The carriers created by interaction of a particle in the crystal are drifted (in first approximation) along the electric fieldlines (electron and holes in opposite direction). The figure 1 show the shape of the electric fieldlines in the crystal if the electrodes are polarized properly.

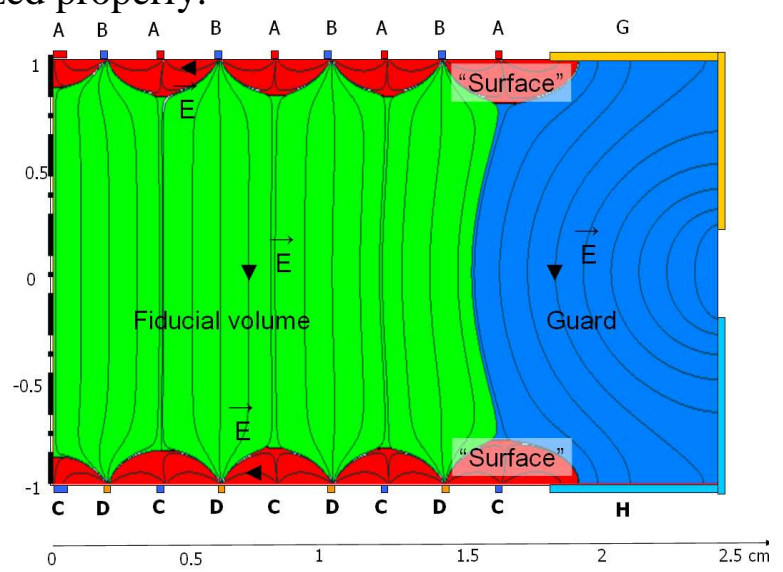

Figure 1: Simulation of the fieldlines (drawn in black) of the detector seen in section (the symmetry axis of the crystal is on the left).

On this figure, the colours of different area of the detector have been chosen as a function of the place of collect of the electron and holes. The green area constitutes the fiducial volume of the detector. An event occurring in this area will deposit electrons on the electrode B and holes on the electrode D and nothing on the electrodes B, C, G and $\mathrm{H}$. The most interest is that, by applying appropriate selection cuts (on the amplitude of the signal read on the electrodes $\mathrm{A}, \mathrm{C}, \mathrm{G}, \mathrm{H}$ ), it is possible to select events from the fiducial volume where the number of surface events has been dramatically reduced.

The figure 2 shows the results of gamma calibration (left) and of low background runs (right) taken with the first prototype of detector with interdigitized electrodes $(200 \mathrm{~g})$ after the fiducial selection. In both figures, the electronic recoil band is filled by gamma event but no event is present in the nuclear recoil band where the WIMP signal ${ }^{2}$ is expected.

Calibrations with beta sources $\left({ }^{210} \mathrm{~Pb}\right)$ have been performed too, demonstrating a high surface event rejection, even at low energy threshold down to $20 \mathrm{keV}$ and below (soon published). 

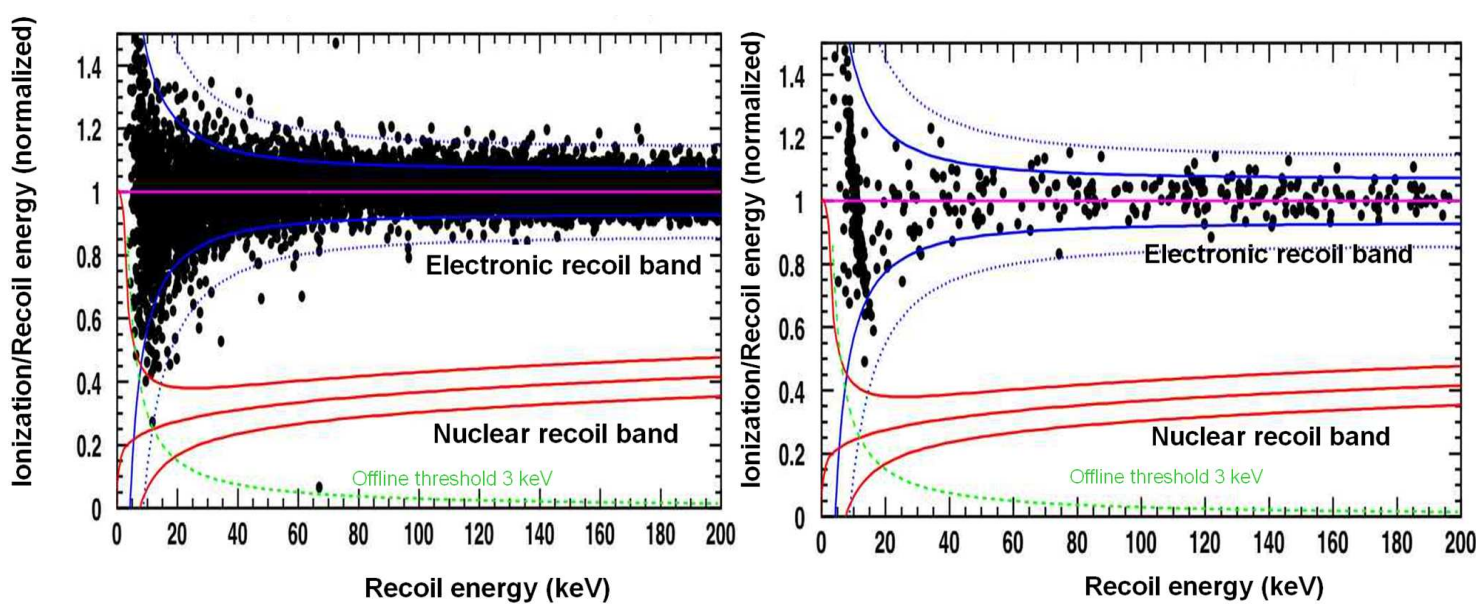

Figure 2: Ratio of the ionization over recoil energy as a function of the recoil energy. Left: for calibration with barium source (gamma of $356 \mathrm{keV}$ ) with a statistics of about 20000 events.

Right: for low background run taken with a detector fitted with interdigitized electrodes corresponding to a total exposure of $4 \mathrm{~kg}$.day.

\section{Conclusion}

In addition to the good surface event rejection, these bolometers offer a relative simplicity of realisation. Moreover their behaviour can be simulated using electric fieldmap. For theese reasons, the EDELWEISS has chosen this type of detector to equip the experiment. For the moment 4 bolometers of this type are running in the EDELWEISS II cryostat, and 9 more will be installed for the end of 2008. In spring 2011 we may have about 60 detectors installed so that we could get a statistic of 3500 kg.day by summer 2012 (corresponding to a sensitivity of a few of $10^{-9} \mathrm{pb}$ of WIMPnucleon cross section).

\section{References}

[1] Fiorucci et al, Astropart. Phys. 28, (2007) 143.

[2] V. Sanglard et al, Phys. Rev. D 71, 122002 (2005).

[3] S. Scorza et al, arXiv:0806.3147v1, astro-ph (2008).

[4] M. Horn, Simulations of the muon-induced neutron background of the EDELWEISS-II experiment for Dark Matter search, Ph.D. thesis, Universitaet Karlsruhe (TH), 2007 and FZKA scientific report 7391 (http://bibliothek.fzk.de/zb/berichte/FZKA7391.pdf)

[5] PCF Di Stefano et al, J Low Temp Physics, 151 (2008) 902.

[6] A. Juillard et al, Nucl. Instrum. Methods, A 559, (2006) 393.

[7] S. Marnieros et al, J.Low Temp. Phys.: Proceedings of the $12^{\text {th }}$ LTD Workshop 2007.

[8] A. Broniatwoski et al, J.Low Temp. Phys.: Proceedings of the $12^{\text {th }}$ LTD Workshop 2007.

[9] X. Defay et al, J.Low Temp. Phys.: Proceedings of the $12^{\text {th }}$ LTD Workshop 2007. 